\title{
Aspectos Perinatales del Embarazo Post-término Complicaciones y Manejo
}

\author{
DEPARTAMENTO DE OBSTETRICIA Y GINECOLOGIA \\ FACULTAD DE SALUD. UNIVERSIDAD DEL VALLE
}

\author{
Dres.: Martha Yelena Echeverry*, Rodrigo Cifuentes B.**
}

El embarazo post-término (EPT) está considerado como una patología de alta morbimortalidad perinatal $(1,2,3)$. Existe un concepto unánime de que el feto que permanece en útero más allá del término de la gestación se encuentra expuesto a un riesgo incrementado por complicaciones inherentes a la prolongación del embarazo por encima de lo normal.

Por otra parte, esta patología obstétrica, además de morbimortalidad perinatal, produce probablemente secuelas de orden sicológico en los padres, mayores que las producidas por otras entidades. El hecho de que los padres esperen un hijo completamente sano en un embarazo que durante los 9 meses ha transcurrido sin problema, y súbitamente se. encuentren con un pobre resultado perinatal, hace pensar que las consecuencias de orden psíquico, pueden ser importantes. Sin embargo, a pesar de todo lo anterior, no existe uniformidad de criterio médico en el manejo obstétrico del $\operatorname{EPT}(4,5,6)$. El presente artículo pretende revisar las complicaciones perinatales más frecuentes de EPT en nuestra población manejada de acuerdo con las conductas del Servicio de Obstetricia y Ginecología del Hospital Universitario del Valle para, al final, tratar de normatizar un manejo adecuado del mismo, tendiente a disminuir la alta morbimortalidad perinatal de esta patología obstétrica.

\section{PACIENTES Y METODOS}

Se estudiaron todas las pacientes que durante el año de 1988 consultaron al Servicio de Obstetri-

* Residente de Segundo Año

** Profesor Titular cia por presentar 42 semanas o más de amenorrea, de acuerdo con la fecha del inicio de su última menstruación (FUM). Del total de pacientes atendidas en este período de tiempo $(\mathrm{n}=9.418$ nacimientos), 762 embarazadas finalizaron su gestación 2 semanas después de la fecha probable (EPP).

Cada una de las pacientes se manejó de acuerdo con el protocolo existente desde hace 2 años en nuestro Servicio de Obstetricia y que, básicamente, consiste en:

1. Amenorrea confiable y cérvix favorable: se induce el trabajo de parto (T de P) a las 42 semanas.

2. Amenorrea confiable, cérvix desfavorable: evaluación del bienestar fetal (ultrasonido obstétrico y monitoría electrónica fetal) e inducción del $\mathrm{T}$ de $\mathrm{P}$ si existe alteración en alguno de ellos (p. ej. oligohidramnios o Prueba de Tolerancia a las Contracciones Uterinas positiva).

3. Amenorrea no confiable: cuidadosa evaluación de la edad fetal y de su medio ambiente, mediante ecografía, monitoría fetal y amniocentesis. La conducta posterior (inducción o no), se decide de acuerdo con los resultados y con el grado de maduración del cuello uterino.

Se revisaron para cada embarazo las siguientes variables:

Maternas: edad, regularidad de los ciclos menstruales, confiabilidad de la amenorrea, edad gestacional al término, y método de finalización del embarazo. 
Feto-neonatales, dependientes del peso: macrosomía, incidencia de cesáreas, desproporción feto pélvica, trauma neonatal (hemorragias intracraneanas).

Feto-neonatales, independientes del peso: aspecto del líquido amniótico, incidencia de sufrimiento fetal, sindrome de aspiración de meconio y sindrome de postmadurez.

Cada una de las anteriores variables se comparó con los resultados obtenidos durante el mismo año en la población obstétrica cuyo embarazo finalizó entre 38 y 41 semanas de amenorrea. Se calculó el riesgo relativo, el Odds Ratio y el Test de Schi cuadrado para establecer dependencia significativa entre la misma variable analizada en las 2 poblaciones diferentes (embarazo post-término y embarazo de término).

\section{RESULTADOS}

Durante el año de 1988, se atendieron 9.418 nacimientos. De esta población, 762 consultaron por amenorrea prolongada, lo cual nos da una incidencia del $8.1 \%$ de Embarazo post-término (EPT). El promedio de edad materna, paridad, amenorrea confiable e incidencia de patología fue sensiblemente igual y no hubo diferencias estadísticamente significativas en ninguno de los parámetros analizados en las 2 poblaciones estudiadas (Tabla 1 ).

TABLA 1

\begin{tabular}{|lrrrr|}
\hline & Post-término & Término & RR & OR \\
\hline Edad materna (X) & 24.4 & 26.2 & 0.81 & 0.80 \\
Analfabetismo & 4.28 & 5.09 & 0.84 & 0.83 \\
Estadó civil estable & 80.57 & 77.42 & 1.04 & 1.21 \\
Hábito de fumar & 7.03 & 7.89 & 0.89 & 0.88 \\
Primigestante & 40.55 & 38.62 & 0.73 & 0.71 \\
Amenorrea confiable & 63.15 & 60.80 & 0.94 & 0.91 \\
Hipertensión crónica & 1.88 & 3.60 & 0.52 & 0.51 \\
Preeclampsia & 8.85 & 16.16 & 0.55 & 0.50 \\
Eclampsia & 0.44 & 0.79 & 0.56 & 0.56 \\
R P M & 13.30 & 20.80 & 0.64 & 0.59 \\
Infección urinaria & 8.73 & 7.74 & 1.13 & 1.14 \\
Otras infecciones & 6.08 & 5.44 & 1.12 & 1.12 \\
Anemia crónica & 0.99 & 0.76 & 1.30 & 1.31 \\
Cardiopatía & 0.44 & 0.55 & 0.81 & 0.81 \\
Parasitosis & 0.66 & 0.28 & 2.38 & 2.38 \\
Mortalidad materna & 0.21 & 0.21 & 1.01 & 1.01 \\
\hline
\end{tabular}

Promedio de edad materna $(\mathrm{X})$ e incidencia porcentual $(\%)$ de las diferentes variables en las 2 poblaciones estudiadas. Fn ninguno de estos parámetros hubo diferencia estadísticamente significativa.

\section{Complicaciones perinatales dependientes del peso}

En las 762 pacientes estudiadas se encontraron 129 con peso mayor de 3.800 grs lo cual da una incidencia de $16.9 \%$ de macrosomía fetal para el EPT. En la población general (PG) esta incidencia fue de $5.1 \%(\mathrm{P}<0.001)$ (Tabla 2$)$. Con peso mayor de 4.000 grs hubo 67 neonatos en el EPT (el $8.8 \%$ mientras que en la PG nacieron 348 neonatos con peso superior a los 4.000 grs el $3.7 \%$ (P < $0.001)$. Sin embargo, la proporción de cesáreas fue sensiblemente igual en ambas poblaciones (25.5 y $24.9 \%$ respectivamente). El diagnóstico de desproporción céfalo pélvica se hizo el doble de veces en el EPT con respecto a la PG: $7.3 \%$ y $4.5 \%$ respectivamente (Tabla 2). Otras complicaciones dependientes del peso fetal como la distocia de hombros y el trauma perinatal mostraron también una incidencia mayor estadísticamente significativa en el embarazo post-término: retención de hombros en el $0.26 \%$ de los fetos, trauma perinatal en el $0.7 \%$ de los neonatos de madres con EP'T. En la PG no hubo ningún caso con distocia de hombros y el trauma perinatal se presentó en el $0.4 \%$ de los recien nacidos (Tabla 2).

TABLA 2

\begin{tabular}{|lccl|}
\hline & Post-término & Término & P \\
\hline Peso neonatal $>3.800$ grs & 16.9 & 5.1 & 0.001 \\
Peso neonatal > 4.000 grs & 8.8 & 3.7 & 0.01 \\
Desproporción céfalo-pélvica & 7.3 & 4.5 & 0.01 \\
desáreas & 25.5 & 24.9 & N. S. \\
Fórceps & 0.8 & 1.6 & N. S. \\
Retención de hombros & 0.26 & 0 & 0.001 \\
Trauma perinatal & 0.72 & 0.44 & 0.01 \\
Hemorragia intracraneana & 0.26 & 0.05 & 0.001 \\
\hline
\end{tabular}

Complicaciones feto-neonatales dependientes del peso en la población con embarazo post-término $(\mathrm{n}=762)$ y en la población general con embarazo a término $(n=9.418)$. Los resultados se presentan en incidencia porcentual para cada variable.

La hemorragia cerebral se diagnosticó en proporción 5 veces mayor en los neonatos productos de embarazo post-término (el $0.26 \%$, de incidencia de hemorragia intracraneana) con relación en los recién nacidos provenientes de madre con embarazo a término (el $0.5 \%$ de incidencia). El riesgo relativo de hemorragia cerebral en los neonatos posttérmino fue de 5.20 (Fig. 1). 
FIGURA 1

\begin{tabular}{|l|c|c|c|c|}
\hline & \multicolumn{4}{c|}{ Hemorragia cerebral } \\
\hline \multirow{3}{*}{\begin{tabular}{l} 
Embarazo \\
\cline { 2 - 5 }
\end{tabular}$>42$ semanas } & SI & $2(0,26 \%)$ & 760 & 762 \\
\cline { 2 - 5 } & NO & $4(0.05 \%)$ & 786.5 & 7.869 \\
\cline { 2 - 5 } & & 6 & 8.625 & 8.631 \\
\hline
\end{tabular}

Incidencia y riesgo relativo de hemorragia cerebral neonatal en el embarazo post-término y en el embarazo a término.

\section{Complicaciones perinatales independientes del peso}

La complicación más frecuente en el EPT fue la presencia de líquido amniótico (L. A.) teñido de meconio: de los 762 nacimientos en 634 se consignó en la historia clínica el aspecto del L.A. De estas 634 , en el $63.8 \%(n=405)$, el L.A. era de aspecto claro y en el $36.1 \%(n=229)$ estaba teñido de meconio. En este grupo con L.A. meconiado, el 10.2\% era meconio espeso y grumoso ("barro meconial") y el $25.8 \%$ correspondía a meconio de color verde y sin grumos.

Cabe destacar que, de los 229 embarazos con L.A. meconiado, 26 (el 11.3\%) hicieron sufrimiento fetal durante el trabajo de parto (diagnosticado por cambios en la frecuencia cardiaca fetal). Mientras que de 405 embarazadas post-término, con L.A. claro, solamente 11 (el 2.7\%), hicieron sufrimiento fetal. Esta diferencia fue estadísticamente significativa (P $<0.001$ Fig. 2). Si se analizan solamente los embarazos con "barro meconial", la incidencia de sufrimiento fetal en este subgrupo, fue de 36.9 (de 65 pacientes con meconio espeso y grumoso, en 24 se hizo el diagnóstico de sufrimiento fetal).

\section{FIGURA 2}

\begin{tabular}{|c|c|c|c|c|c|c|}
\hline \multicolumn{7}{|c|}{ Sufrimiento fetal } \\
\hline \multirow{4}{*}{$\begin{array}{l}\text { L.A. } \\
\text { Meconiado }\end{array}$} & & SI & No & & \multirow{4}{*}{$\begin{array}{l}\mathrm{X}^{2}= \\
\mathrm{P}<\end{array}$} & \\
\hline & SI & $26(11.3 \%)$ & 203 & 229 & & 19.9 \\
\hline & No & $11(2.7 \%)$ & 394 & 405 & & 0.001 \\
\hline & & 37 & 597 & 634 & & \\
\hline
\end{tabular}

Relación de dependencia estadística entre presencia de meconio en el líquido amniótico y sufrimiento fetal (Diagnosticado por cambios en la frecuencia cardíaca fetal).
En 31 pacientes de la población con EPT se hizo diagnóstico ecográfico de disminución en el volumen del L.A., lo cual nos da una incidencia del $4.06 \%$ de oligohidramnios para la población estudiada por nosotros. El sindrome de post-madurez caracterizado fundamentalmente por retardo en el crecimiento intrauterino con disminución en la grasa subcutánea, uñas y cabellos largos, facies expresiva e impregnación de meconio en la piel del neonato, se encontró solamente en 26 recién nacidos, de los 762 estudiados, lo cual nos da una incidencia de este sindrome del $3.41 \%$ en el EPT (Tabla 3).

El Sindrome de Aspiración de Meconio se encontró solamente en 16 neonatos de post-término (el $2.10 \%$ de los embarazos prolongados), mientras que en los embarazos de término, este diagnóstico se hizo en el $1.44 \%$ de los neonatos (P H 0.05).

En las pacientes con embarazo post-término, hubo 11 muertes fetales en las cuales 9 (el 81.8\%) tenían el L.A. teñido de meconio, un anencéfalo y uno con malformaciones congénitas múltiples. Lo anterior da una mortalidad fetal en la población con EPT del 14 por mil, mientras que en la población muestra, con embarazo entre 38 y 41 semanas, la mortalidad fetal fue de 42 por mil.

La mortalidad neonatal en las dos poblaciones estudiadas fue del 4 por mil en el EPT y del 24 por mil en el embarazo a término. La incidencia de malformaciones congénitas fue del $1.8 \%$ en los neonatos post-término, mientras que en los neonatos a término se presentó el $2.16 \%$ de malformaciones. Las malformaciones congénitas en los productos del EPT fueron las siguientes: Anencefalia (1), macrocefalia (1), meningocele (1), espina bífida (3), agenesia renal (3), polidactilia (1) y malformaciones múltiples (4).

\section{CONCLUSIONES}

El embarazo post-término en nuestra población tiene una incidencia bastante alta: el $8.1 \%$, ocupando un destacado lugar en la patología obstétrica. Analizadas las complicaciones más frecuentes en esta población, podemos concluir que la macrosomía fetal y el paso de meconio al líquido amniótico, son las principales complicaciones en este grupo de embarazadas, como quiera que esas 2 patologías determinan una serie de alteraciones fetoneonatales productoras de casi toda la morbilidad perinatal en el EPT. Vale decir que con el protocolo nuestro de manejo ya mencionado (4), evitamos la mortalidad feto-neonatal, pero no gran parte de la 
TABLA 3

\section{COMPLICACIONES FETO NEONATALES INDEPENDIENTES DEL PESO EN LA POBLACION GENERAL CON EMBARAZO A TERMINO Y POST-TERMINO (INCIDENGIAS PORCENTUALES).}

\begin{tabular}{|lcccc|}
\hline & Post-término & Término & RR & P \\
\hline Sindrome de post-madurez & 3.41 & - & - & - \\
Sindrome aspiración de meconio & 2.10 & 1.44 & 1.20 & 0.05 \\
Apgar de 6 menos, al ler, minuto & 11.7 & 11.03 & 0.78 & N.S. \\
Apgar de 6 o menos, al 5o. minuto & 0.5 & 0.79 & 0.95 & N.S. \\
Malformaciones congénitas & 1.80 & 2.16 & 0.83 & N.S. \\
Mortalidad fetal & 1.6 & 4.6 & 0.34 & N.S. \\
Mortalidad neonatal & 0.42 & 2.41 & 0.18 & N.S. \\
\hline
\end{tabular}

morbilidad. En consecuencia, se impone modificar el manejo de esta población obstétrica, adoptando unas normas tendientes a prevenir las complicaciones asociadas al embarazo post-término. Entre esas normas, la mayoría ya mencionadas por otros autores (7), se podrían adoptar como patrones de conducta, las siguientes:

1. En la primera consulta prenatal, la cual debe ser idealmente en la primera mitad del embarazo, definir lo más exactamente posible, la edad gestacional. Para ello, debe precisarse claramente la fecha de la última menstruación (FUM) y establecer su confiabilidad o no. Pensamos que el examen por ultrasonido del feto, es de gran ayuda diagnóstica y debe hacerse en todos los embarazos entre 18 y 22 semanas. Si la ecografía coincide con la FUM, así ésta sea "no confiable", pasa a tener valor en cuanto a confiabilidad para asignar edad gestacional.

2. Una vez definida la edad gestacional se debe calcular la fecha probable del parto (FPP), en lo posible, repetimos, en la primera mitad del embarazo. Esta FPP (40 semanas), no debe modificarse con el transcurso de la gestación.

3. Una vez que se llegue a la FPP, si el cérvix es favorable para una inducción, se debe finalizar el embarazo, máximo a las 41 semanas. Es decir, si el cérvix tiene una posición central con dilatación al menos de $2 \mathrm{cms}$ y borramiento de 50 por ciento, debe inducirse el T. de P. Pensamos que si el feto ha alcanzado la madurez suficiente, el hecho de permanecer en el útero le va a hacer aumentar de peso, además de aumentar las probabilidades de eyección de meconio en el L.A. En consecuencia, ello se podría evitar induciendo los embarazos a término que presentan un cérvix bien favorable para una inducción. Somos conscientes que el adoptar esta conducta puede traer un incremento de intervenciones cesáreas, pero creemos también que ese incremento se daría solamente en casos en que el obstetra decide hacer inducciones con cérvix inadecuado. Cuando la inducción se hace con un cérvix bien maduro, las probabilidades de éxito son cercanas al ciento por ciento.

4. Si no se finaliza el embarazo (por cérvix inadecuado), debe hacerse una cuidadosa evaluación ecográfica del peso fetal. Si éste se calcula en más de 4.000 grs, el embarazo, es preferible, finalizarlo con operación cesárea.

5. Finalmente, insistir en que parte integral del manejo del EPT es la evaluación seriada del medio ambiente fetal a través del ultrasonido y la monitoría electrónica fetal para detectar cambios iniciales en el estado de vitalidad fetal (oligohidramnios o Prueba de Tolerancia a las Contracciones, positiva). Quizá este manejo es el que nos ha permitido tener una mortalidad fetal en el EPT inferior a la registrada en el mismo tiempo en la población con embarazo a término. En consecuencia, los esfuerzos en el manejo futuro del EPT deben dirigirse a disminuir la alta morbilidad producida por la macrosomía fetal y por la eyección de meconio en el L.A. Pensamos que las conductas aquí sugeridas, pueden contribuir a la solución del problema del embarazo post-término. 